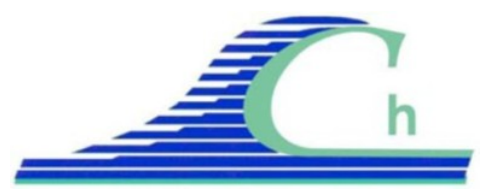

XII ${ }^{\text {èmes }}$ Journées Nationales Génie Côtier - Génie Civil

Cherbourg, 12-14 juin 2012

DOI:10.5150/jngcgc.2012.005-D @ Editions Paralia CFL

disponible en ligne - http://www.paralia.fr - available online

\title{
Interactions vagues-courants dans une embouchure tidale dominée par la houle
}

\author{
Guillaume DODET $^{1,2}$, Xavier BERTIN ${ }^{1}$, Nicolas BRUNEAU ${ }^{2,3}$, \\ André FORTUNATO ${ }^{2}$, Alphonse NAHON ${ }^{2}$, Rui TABORDA ${ }^{4}$
}

1. Institut du Littoral et de l'Environnement, UMR 7266 CNRS - Université de La Rochelle, 2 rue Olympe de Gouges, 17000 La Rochelle Cedex, France.

guillaume.dodet@univ-lr.fr

2. Division des Estuaires et Zones Côtières, Laboratoire National d'Ingénierie Civile, Avenida do Brasil, 101, Lisbonne 1700-066, Portugal.

gdodet@Inec.pt

3. British Antarctic Survey, High Cross, Madingley Road, Cambridge, CB30ET, UK.

4. Faculté des Sciences de l’Université de Lisbonne, LATTEX, IDL, Campo Grande, 1749-016 Lisbonne, Portugal.

\section{Résumé :}

Les embouchures tidales permettent les échanges d'eau et de sédiments entre l’océan et une lagune séparée de celui-ci par un cordon littoral. L’hydrodynamique de ces embouchures est contrôlée par la propagation des vagues et de la marée qui, dans le cas de systèmes peu profonds, peut entraîner des évolutions morphologiques rapides. Une meilleure compréhension du forçage hydrodynamique est donc nécessaire pour pouvoir anticiper ces évolutions. Cette étude présente une application d'un système de modélisation numérique (SMN), couplant un modèle de vagues avec un modèle de circulation hydrodynamique, à la lagune d'Albufeira au Portugal. La comparaison des résultats du SMN avec des données hydrodynamiques in situ a permis d'évaluer les performances de ce SMN. L'application de différentes configurations du SMN (sans vague, avec vagues et avec rétroaction des courants sur les vagues) a ensuite permis d'étudier l'effet des vagues sur la circulation et la rétroaction des courants sur le champ de vagues incident. Les résultats montrent que la contribution du setup induit par les vagues sur l'élévation de la surface libre peut atteindre jusqu'à $30 \mathrm{~cm}$ dans le chenal (pour des vagues de $1.5 \mathrm{~m}$ au large). De plus, lors du jusant, la hauteur significative des vagues augmente fortement en face de l'embouchure et diminue rapidement en se propageant dans le chenal. La réfraction ainsi que le blocage des vagues par les courants semblent être responsables de cette modification du champ de vagues.

Mots-clés:

Hydrodynamique côtière - Modélisation numérique - Blocage des vagues 


\section{Introduction}

Les systèmes lagunaires et leurs embouchures sont des niches écologiques et touristiques dont l'équilibre est conditionné par la qualité de l'eau et la stabilité du trait de côte. Une connaissance approfondie de l'hydrodynamique et de la dynamique sédimentaire de ces systèmes est nécessaire afin d'anticiper leurs évolutions morphologiques et mettre en place un système de gestion adéquat et durable.

Les premiers travaux sur les embouchures tidales ont permis d'établir des relations entre plusieurs caractéristiques telles que l'amplitude de la marée, le prisme tidal, la surface de la section mouillée ou encore la hauteur des vagues incidentes. Ces travaux ont donné lieu à des théories empiriques et conceptuelles ainsi qu'à un classement des différents types d'embouchures encore largement utilisées (HAYES, 1979 ; VINCENT \& CORSON, 1980). D'autres auteurs ont utilisé des approches simplifiées d'hydraulique en écoulement stationnaire pour formuler des solutions analytiques permettant d'étudier les écoulements dans le chenal et la modulation tidale dans la lagune (KEULEGAN, 1967 ; MOTA OLIVEIRA, 1970). Plus récemment, des modèles numériques ont été appliqués à différents types d'embouchure tidale, dominée par la marée (CAYOCCA, 2001) ou bien par les vagues (RANASINGHE et al., 1999 ; BERTIN et al., 2009 ; BRUNEAU et al., 2011), afin d'obtenir des informations plus détaillées sur les principaux processus régissant la circulation et la dynamique sédimentaire de ces systèmes.

L'hydrodynamique des embouchures tidales est contrôlée principalement par l'action combinée de la marée et des vagues. L'effet des vagues sur la circulation horizontale a été formulé par LONGUET-HIGGINS \& STEWART (1964). PHILLIPS (1977) proposa une formulation de l'équation de conservation de la densité spectro-angulaire d'action des vagues, incluant les courants. Des études expérimentales ont permis d'étudier les effets des courants sur les vagues, en particulier le phénomène de blocage des vagues par un courant contraire (LAI et al., 1989 ; CHAWLA \& KIRBY, 2002) . Ce phénomène reste encore très mal compris et peu d'études en présentent des observations en milieu naturel (e.g. WOLF \& PRANDLE, 1999).

Dans cette étude, un système de modélisation numérique (SMN) est mis en place afin d'étudier les interactions vagues-courants dans une embouchure tidale. Les résultats du modèle sont d'abord comparés à des données hydrodynamiques mesurées dans une embouchure tidale de la côte ouest portugaise. Le modèle est ensuite utilisé pour étudier la contribution du setup induit par les vagues sur l'élévation de la surface libre ainsi que les effets des courants de jusant sur le champ de vagues incident. 


\section{XII ${ }^{\text {èmes }}$ Journées Nationales Génie Côtier - Génie Civil \\ Cherbourg, 12-14 juin 2012}

\section{Caractérisation du site d'étude}

\subsection{Contexte géomorphologique}

La lagune d'Albufeira se situe sur la côte ouest portugaise, à $20 \mathrm{~km}$ au sud de Lisbonne (figure 1). La largeur du plateau continental le long de l'arc littoral Caparica-Espichel est comprise entre $30 \mathrm{~km}$, en face de l'embouchure du Tage, et $5 \mathrm{~km}$ en face de la lagune où le canyon de Lisbonne intersecte le plateau. La lagune couvre une surface allongée de $1.3 \mathrm{~km}^{2}$, légèrement oblique (SO-NE) par rapport à la côte (N-S). Elle est composée de deux corps lagunaires principaux : la petite et peu profonde ( $\sim 5 \mathrm{~m}$ ) Lagoa Pequena située à l'extrémité Est et la plus étendue et plus profonde ( 15 m) Lagoa Grande (notées "LP" et "LG" sur la figure 1). Cette dernière est connectée à l'Océan Atlantique par un chenal étroit (quelques dizaines de mètres de large) et peu profond (quelques mètres au plus). La morphologie du chenal présente un comportement saisonnier qui conduit de manière quasi-systématique à son élargissement pendant l'été et son comblement au début de l'hiver. Au printemps, une brèche est créée artificiellement dans le cordon littoral pour permettre le renouvellement des eaux lagunaires.

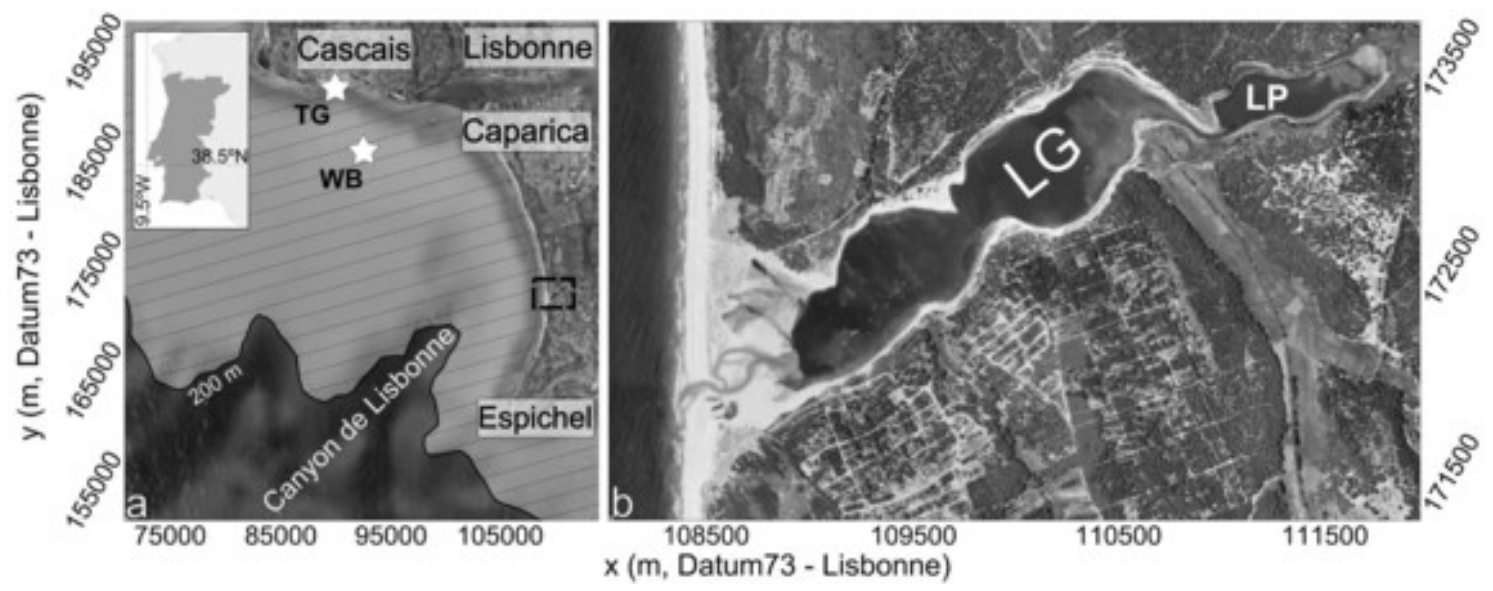

Figure 1. Situation géographique du site d'étude, plateau continental (zone hachurée, corps lagunaires (LG et LP) et positions du marégraphe de Cascais (TG) et du houlographe de Lisbonne (WB).

\subsection{Contexte hydrodynamique}

La côte ouest portugaise est soumise à un régime de marée de type meso-tidal, l'amplitude moyenne de la marée étant comprise entre $1 \mathrm{~m}$ et $3.5 \mathrm{~m}$. Lorsque la marée se propage dans la lagune, l'amplitude des constituants semi-diurnes est atténuée alors que les constituants non-linéaires quart-diurnes et bimensuels sont amplifiés (MEHTA \& ÖZSÖY, 1978), donnant lieu à un signal de marée fortement asymétrique. 
Le climat de vagues est dominé par des houles de longues périodes, de secteur de provenance nord-ouest, générées dans l’océan Atlantique Nord. Une simulation rétrospective de 57 ans de paramètres moyens de houle dans l'Atlantique Nord-Est (DODET et al., 2010) révèle des moyennes annuelles de $1.9 \mathrm{~m}$, et $10.5 \mathrm{~s}$ pour la hauteur significative (HS) et la période de pic (TP), à $100 \mathrm{~km}$ au large de la lagune. Les moyennes hivernales correspondantes sont de $2.5 \mathrm{~m}$ et $12.1 \mathrm{~s}$ et attestent du caractère énergétique de la houle dans cette région.

\subsection{La campagne de mesures}

Une campagne de mesures a eu lieu du 23 au 24 septembre 2010. Deux capteurs de pressions (PT; Level TROLL 500 - In-situ Inc.), deux courantomètres électromagnétiques (ECM; INFINITY-EM - JFE Advantech Co., Ltd) et un courantomètre à effet Doppler avec capteur de pression intégré (ADCP; Aquadopp Profiler $2 \mathrm{MHz}$ - Nortek AS) ont été installés pendant la première marée basse du 23 ( 9h UTC). La topographie du site d'étude a été mesurée au moyen de GPS (RTK et différentiel) et les données manquantes de la zone intertidale ont été complétées par des mesures bathymétriques réalisées par échosondeur embarqué en mars 2010. La figure 2 montre la bathymétrie du site d'étude ainsi que la position des instruments utilisés lors de cette campagne.

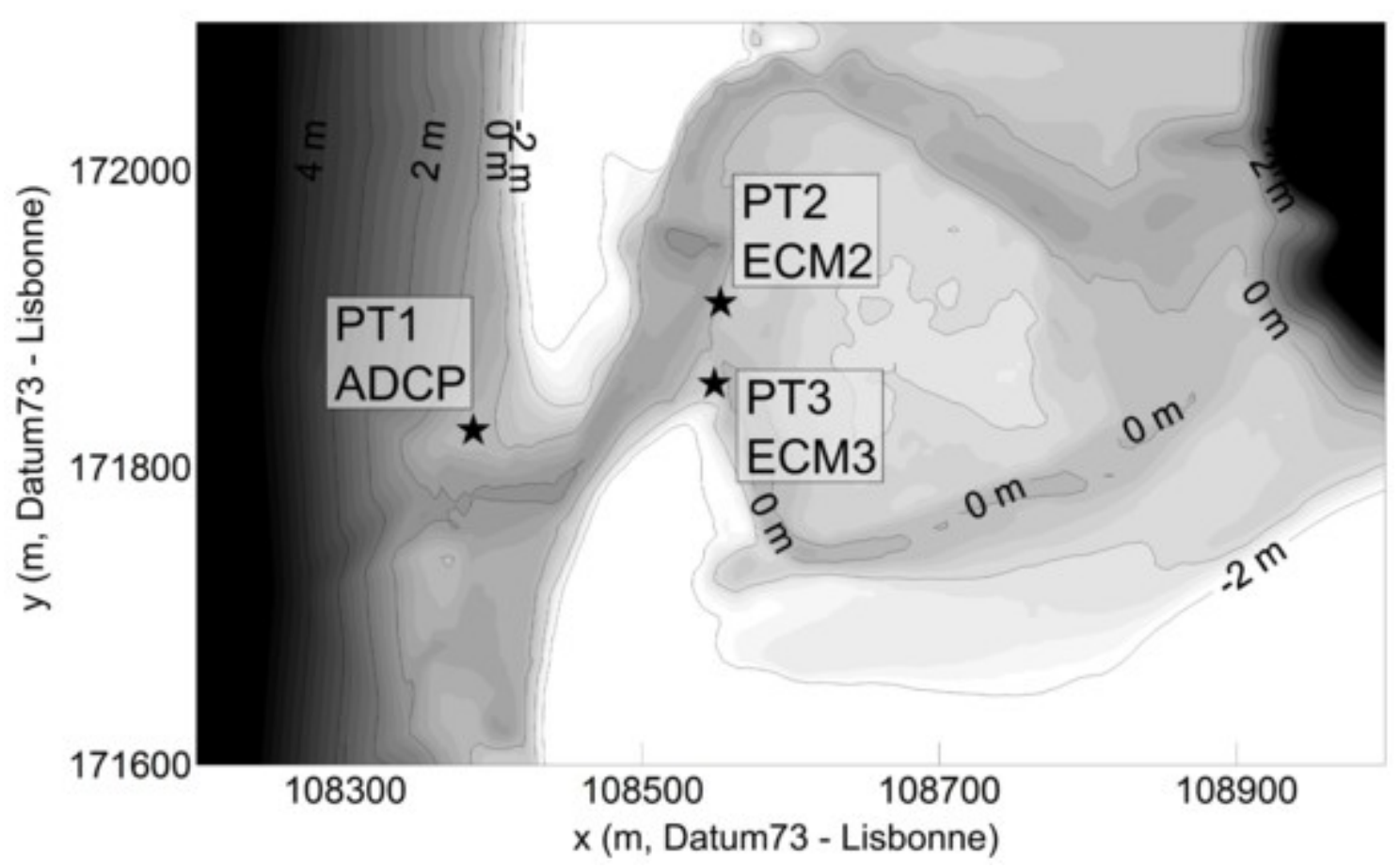

Figure 2. Bathymétrie et position des capteurs (PT1-ADCP: $15 \mathrm{~cm}$ * PT2-ECM2: 26 cm*; PT3-ECM3: $50 \mathrm{~cm} *$; * au-dessus du niveau moyen). 


\section{XII ${ }^{\text {èmes }}$ Journées Nationales Génie Côtier - Génie Civil \\ Cherbourg, 12-14 juin 2012}

\section{Le système de modélisation vague-courant}

\subsection{Description du modèle}

Le SMN utilisé pour cette étude a été développé en vue de simuler la circulation et la dynamique des sédiments non cohésifs en milieu estuarien sous l'action de la marée et des vagues. Le noyau de ce SMN est le modèle de circulation SELFE (ZHANG \& BAPTISTA, 2008) qui résout les équations de Navier-Stokes à deux (2DH) ou trois dimensions (3D) et les équations de transport pour la salinité et la chaleur. Ce modèle, utilisé pour notre étude dans sa version 2DH, a été couplé au modèle de vagues SWAN (BOOIJ et al., 1999) qui résout l'équation de conservation de densité spectro-angulaire d'action des vagues. Le couplage est externe (le pas de temps du modèle de circulation est différent de celui du modèle de vague) et total (échange d'informations dans les deux sens). Une grille de calcul non structurée (à éléments triangulaires) de 28000 nœuds est utilisée pour le modèle de circulation. Un signal de marée reconstitué à partir de 16 constituants (O1, K1, P1, Q1, M2, S2, N2, K2, 2N2, Mu2, Nu2, M3, M4, MS4, MN4, M6) est imposé à la frontière. Une formule quadratique du frottement au fond est utilisée avec un coefficient de Manning de $0.04 \mathrm{~s} . \mathrm{m}^{-1 / 3}$. La propagation des vagues se fait par l'intermédiaire de trois grilles de calcul emboitées : les deux premières étant régulières et de résolutions respectives $1000 \mathrm{~m}$ et $200 \mathrm{~m}$; la troisième grille est rectilinéaire et sa résolution est comprise entre $90 \mathrm{~m}$ au large et $2.5 \mathrm{~m}$ dans le chenal et en zone de déferlement. Ces trois grilles comportent 8000 nœuds au total et sont forcées par les sorties spectrales d'un modèle de vagues dont l'emprise géographique recouvre l’océan Atlantique Nord.

\subsection{Validation du modèle}

Les résultats du modèle ont été comparés en premier lieu aux données mesurées au large de l'embouchure afin de valider les conditions aux frontières du SMN. L'élévation de la surface libre mesurée au marégraphe de Cascais est reproduite avec une erreur quadratique moyenne (EQM) de $1.9 \mathrm{~cm}$ alors que les HS et TP mesurées au large de l'embouchure du Tage sont reproduites avec des EQM respectives de $29.3 \mathrm{~cm}$ et $1.8 \mathrm{~s}$ (soit $17.6 \%$ et $15.9 \%$ une fois normalisées par la moyenne des données, plus loin noté EQMN).

Les comparaisons entre les HS prédites par le modèle et celles mesurées à la position des trois PT (figure 3) donnent des EQMN moyennes de $42.0 \%$ dans le cas de la simulation sans rétroaction des courants sur les vagues et de $30.3 \%$ dans le cas de la simulation avec rétroaction des courants sur les vagues.

Concernant l'élévation de la surface libre, les comparaisons donnent des EQM moyennes de $12.1 \mathrm{~cm}$ dans le cas de la simulation sans les vagues et de $6.9 \mathrm{~cm}$ dans le cas de la simulation avec les vagues (figure 4). 
Pour valider les vitesses prédites par le SMN, seules les données mesurées par l'ECM2 ont pu être exploitées, les autres étant erronées à cause du déferlement des vagues dans le cas de l'ADCP et à cause de l'enfouissement de l'ECM3. Les comparaisons donnent une EQMN de $21.2 \%$ pour la norme de la vitesse dans le cas des simulations avec vagues (avec ou sans rétroaction des courants).

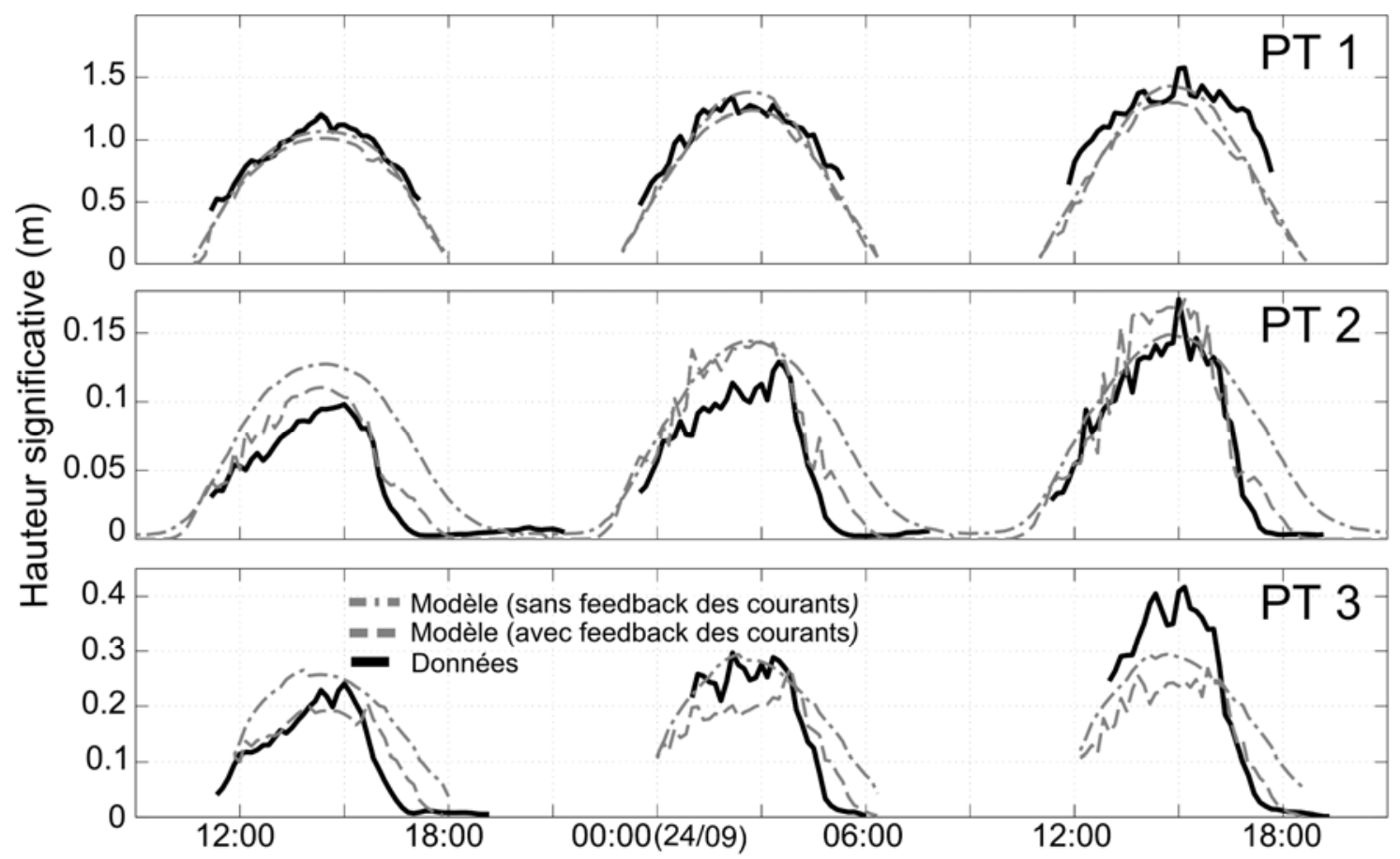

Figure 3. Comparaison des HS mesurées (pointillés) et simulées.

\section{Discussion - Conclusion}

\subsection{Effet des vagues sur la circulation}

En déferlant dans les zones peu profondes du rivage, les vagues dissipent une partie de leur énergie et transfèrent la quantité de mouvement qu'elles véhiculent à la colonne d'eau. Ce phénomène entraîne une inclinaison du plan d'eau qui permet d'équilibrer la composante onshore du flux de quantité de mouvement. Il en résulte une surcote de l'élévation de la surface libre dans la zone de déferlement, et dans le chenal et la lagune. Les résultats du modèle montrent que le SMN est capable de reproduire cette surcote, ce qui permet d'améliorer les prédictions de l'élévation de la surface libre dans la lagune. Cette surcote, qui peut atteindre jusqu'à $30 \mathrm{~cm}$ dans le chenal (avec des vagues de $1.5 \mathrm{~m}$ au large), nécessite d’être prise en compte dans le calcul du transport sédimentaire. 


\section{XII ${ }^{\text {èmes }}$ Journées Nationales Génie Côtier - Génie Civil \\ Cherbourg, 12-14 juin 2012}

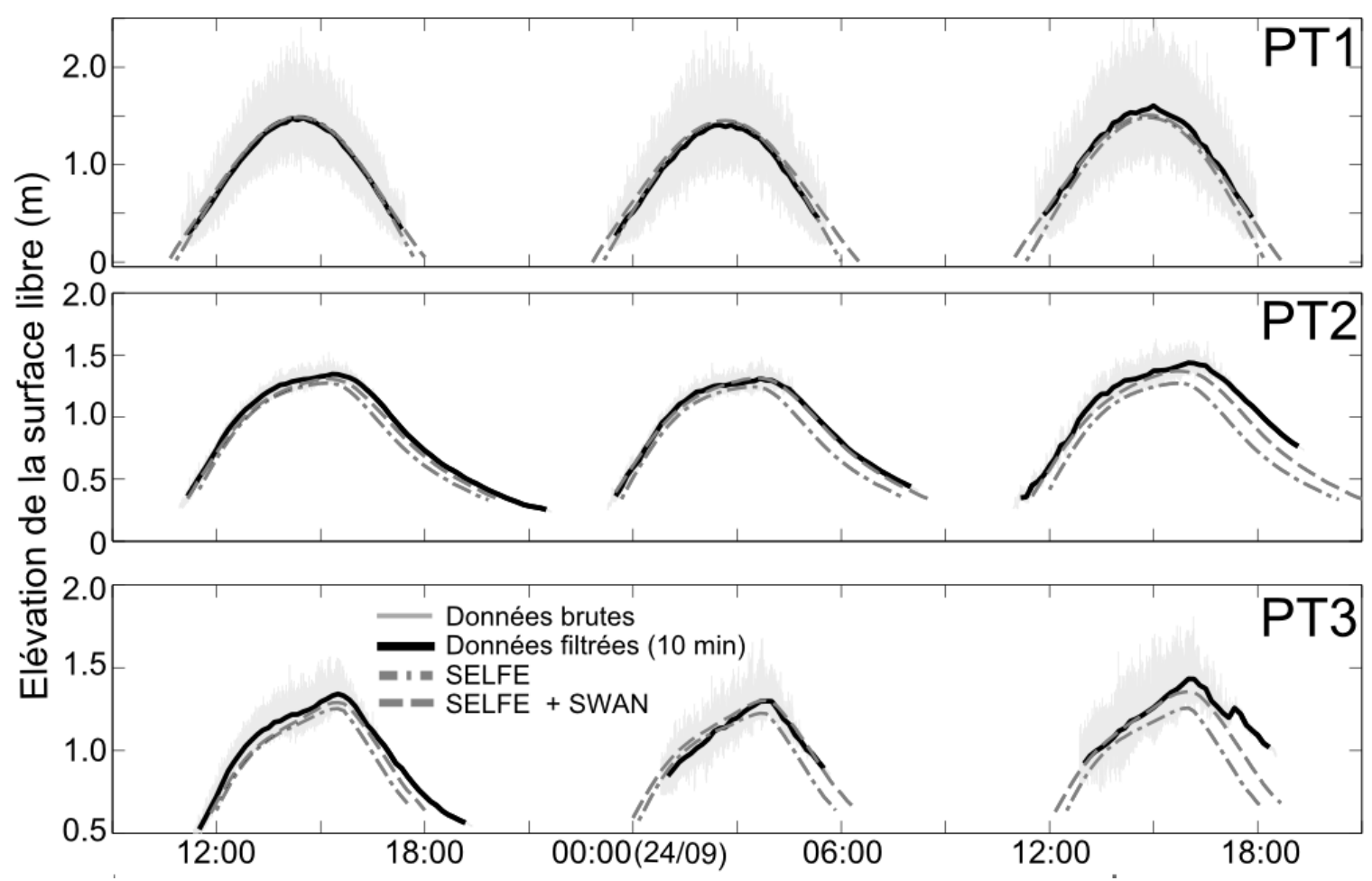

Figure 4. Comparaison des élévations de la surface libre mesurées (pointillés) et simulées.

\subsection{Effet du courant de jusant sur la houle incidente}

En présence du courant de jusant (courant contraire à la direction de propagation des vagues, en première approximation), le champ de vagues est modifié de plusieurs manières, dont les principales sont : (1) le front d'onde est réfracté et sa direction de propagation tend à s'orienter selon l'axe du courant; (2) la fréquence intrinsèque (relative) diminue par effet Doppler ; (3) la hauteur des vagues augmente en réponse à la diminution de la longueur d'onde par conservation de la densité d'action des vagues. Quand la vitesse du courant approche la vitesse de phase des vagues, la propagation de l'énergie des vagues est bloquée et la hauteur des vagues chute brutalement. Ce phénomène est visible sur la figure 3 pour les capteurs PT2 et PT3 qui présentent une diminution rapide de la HS pour les signaux mesuré et simulé avec la rétroaction des courants sur les vagues activée (comparaison figure $5 \mathrm{~d} / 5 \mathrm{~h}$ ).

Afin d'étudier l'effet des courants de jusant sur la propagation des vagues dans l'embouchure, un cas synthétique représentatif des conditions de tempête hivernale (définies ici comme la HS correspondant au $95^{\mathrm{e}}$ centile annuel et la direction et la période associées, soit $4.0 \mathrm{~m}, 302.7^{\circ}$ et $11.2 \mathrm{~s}$ au large, respectivement) a été simulé, dans deux configurations différentes : avec ou sans rétroaction des courants. Le signal de marée est représenté par un seul constituant (M2), dont l'amplitude est fixée à $1.5 \mathrm{~m}$, ce qui correspond à un marnage de vive-eau. Les cartes de courants fournis en entrée du 
modèle de vagues $(\mathrm{U})$, de vitesses de groupe $\left(\mathrm{C}_{\mathrm{g}}\right)$, de somme des vitesses de courants et vitesses de groupe $\left(\mathrm{U}+\mathrm{C}_{\mathrm{g}}\right)$ et de HS simulés pour les deux configurations lors du jusant ( $2 \mathrm{~h}$ après l'étale de marée haute océanique) montrent que la HS est amplifiée à l'entrée du chenal entre les deltas de jusant (zone foncée en bas gauche de la figure $5 \mathrm{~h}$ ) et fortement atténuée à l'intérieur du chenal, dans la zone où U et $\mathrm{Cg}$ tendent à s'annuler (figure 5). La HS simulée dans la configuration avec courants peut être localement $60 \%$ inférieure à celle prédite sans rétroaction des courants.
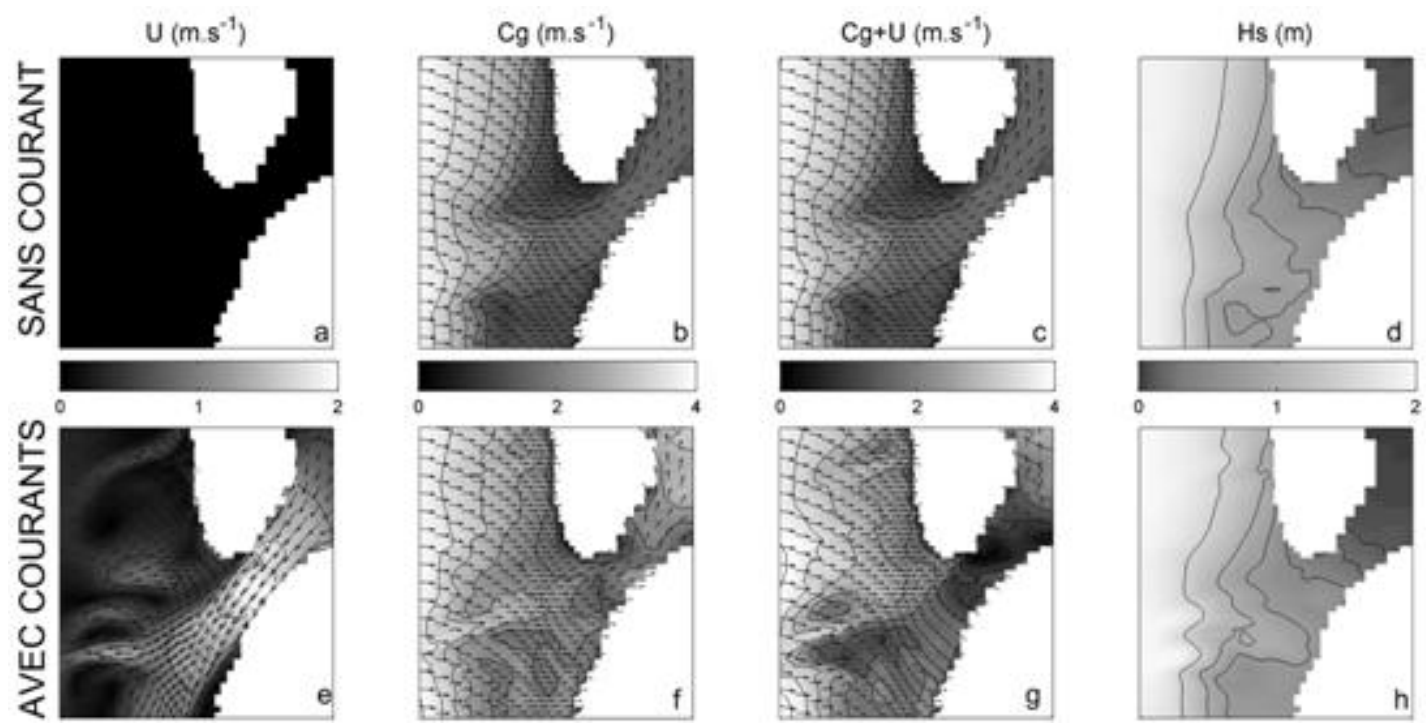

Figure 5. Carte de champs de vitesses de courants fournis en entrée du modèle de vague ( $U$ ), vitesses de groupe des vagues $(\mathrm{Cg})$, somme des vitesses de courants et vitesses de groupe $(U+C g)$ et HS simulés dans le cas de simulations avec rétroaction des courants sur les vagues activée (en bas) ou non (en haut).

\subsection{Conclusion}

Cette étude montre que le SMN développé reproduit l'hydrodynamique d'une embouchure tidale dominée par la houle de manière satisfaisante. Les résultats ont confirmé l'importance de l'effet des vagues sur la circulation afin de reproduire fidèlement l'élévation de la surface libre dans la lagune. Il est aussi montré que les courants ont une influence significative sur la propagation du champ de vagues et qu'il semble nécessaire d'activer cette rétroaction dans le modèle de vagues afin de prédire les paramètres moyens de vagues plus précisément. Ces résultats sur les interactions vagues-courants seront particulièrement importants lors de l'évaluation des performances du module morphodynamique de ce SMN.

\section{Remerciements}

Cette étude est financée par la Fondation portugaise pour la Recherche et la Technologie (FCT) dans le cadre du projet de recherche MOWADI 


\section{XII ${ }^{\text {èmes }}$ Journées Nationales Génie Côtier - Génie Civil \\ Cherbourg, 12-14 juin 2012}

(PTDC/ECM/103801/2008). Le troisième auteur a été financé en partie par la FCT (SFRH/BPD/67041/2009). La campagne de mesure a impliqué de nombreuses personnes du Laboratoire Nationale d’Ingénierie Civile de Lisbonne, de la Faculté des Sciences de l'Université de Lisbonne et de l'Université d'Algarve qu'il convient de remercier.

\section{Références bibliographiques}

BERTIN X., FORTUNATO A.B., OLIVEIRA A. (2009). A modeling-based analysis of processes driving wave-dominated inlets. Continental Shelf Research, Vol. 29 (5-6), pp 819-834. doi:10.1016/j.cs.2008.12.019

BOOIJ N., RIS R., HOLTHUIJSEN L. (1999). A third-generation wave model for coastal regions. 1. Model description and validation. Journal of Geophysical Research, Vol. 104 (C4), pp 7649-7666. doi:10.1029/98JC02622

BRUNEAU N., FORTUNATO A.B., DODET G., FREIRE P., OLIVEIRA A., BERTIN $\mathrm{X}$. (2011). Future evolution of a tidal inlet due to changes in wave climate, sea level and lagoon morphology (Óbidos Lagoon, Portugal). Continental Shelf Research, Vol. 31 (18), pp 1915-1930. doi:10.1016/j.csr.2011.09.001

CAYOCCA F. (2001). Long-term morphological modeling of a tidal inlet: the Arcachon Basin, France. Coastal Engineering, Vol. 42 (2), pp 115-142. doi:10.1016/503783839(00)00053-3

CHAWLA A., KIRBY J.T. (2002). Monochromatic and random wave breaking at blocking points. Journal of Geophysical Research, Vol. 107, pp. 4-1- 4-19. doi:10.1029/2001JC001042

DODET G., BERTIN X., TABORDA R. (2010). Wave climate variability in the NorthEast Atlantic Ocean over the last six decades. Ocean Modelling, Vol. 31, pp 120-131. doi:10.1016/j.ocemod.2009.10.010

HAYES M.O. (1979). Barrier Island morphology as a function of tidal and wave regime. Leatherman (Ed.), Barrier Island. Academic Press, New York, pp 1-28.

KEULEGAN G.H. (1967). Tidal Flow in Entrances: Water Level Fluctuations of Basins in Communication with the Seas. Committee on Tidal Hydraulics Technical Bulletin No.14, U.S. Army Engineers Waterways Experiment Station, Vicksburg, MS. LAI R.J., LONG S.R., HUANG N.E. (1989). Laboratory studies of wave-current interaction: kinematics of the strong interaction. Journal of Geophysical Research, Vol. 94, n ${ }^{\circ}$ C11, pp 16,201-16,214. doi:10.1029/JC094iC11p16201

LONGUET-HIGGINS M.S., STEWART R.W. (1964). Radiation stresses in water waves: a physical discussion with applications. Deep Sea Research, Vol. 11, pp 529-562.

MEHTA A.J. ,ÖZSÖY E. (1978). Inlet hydraulics. In: Bruun,P. (Ed.), Stability of Tidal Inlets: Theory and Engineering. Elsevier Scientific Publishing Co., Amsterdam, The Netherlands, pp 83-161. 
Thème 1 - Hydrodynamique côtière

MOTA OLIVEIRA I.B. (1970). Natural flushing ability in tidal inlets. $12^{\text {th }}$ Coastal Engineering Conference, ASCE, pp 1827-1845.

PHILLIPS O.M. (1977). The dynamics of the upper ocean. Cambridge University Press. RANASINGHE R., PATTIARATCHI C., MASSELINK G. (1999). A morphodynamic model to simulate the seasonal closure of tidal inlets. Coastal Engineering, Vol. 37 (1), pp 1-36. doi:10.1016/50378-3839(99)00008-3

VINCENT C.L., CORSON W.D. (1980). The Geometry of Selected US Tidal Inlets. GITI Report n²0, U.S. Army Corps of Engineers, Washington, DC.

WOLF J., PRANDLE D. (1999). Some observations of wave-current interaction. Coastal Engineering, Vol. 37, pp 471-485. doi:10.1016/50378-3839(99)00039-3

ZHANG Y.-L., BAPTISTA A.M. (2008). SELFE: A semi-implicit Eulerian-Lagrangian finite-element model for cross-scale ocean circulation. Ocean Modelling, Vol. 21 (3-4), pp 71-96. doi:10.1016/j.ocemod.2007.11.005 